\title{
Immunoglobulin $G$ testing in the diagnosis of food allergy and intolerance
}

\author{
TH Lee *, ${ }^{1}$ ScD(Cantab), FRCP(UK), YY Wu, ${ }^{2}$ MB, ChB, DABA\&l, June KC Chan, ${ }^{1}$ RD (USA), MSc, HK Ho, ${ }^{3}$ MD (HKU), FRCPCH, \\ Philip H Li, ${ }^{4} \mathrm{MB}, \mathrm{BS}, \mathrm{MRCP}$, Jaime SD Rosa Duque, ${ }^{3,4} \mathrm{MD}$, PhD; for the Hong Kong Institute of Allergy

\begin{abstract}
${ }^{1}$ Allergy Centre, Hong Kong Sanatorium \& Hospital, Happy Valley; ${ }^{2}$ Centre for Allergy and Asthma Care, Central; ${ }^{3}$ Department of Immunology, Department of Medicine, Queen Mary Hospital, The University of Hong Kong; Hong Kong
\end{abstract} \\ Paediatrics and Adolescent Medicine, Queen Mary Hospital, The University of Hong Kong; ${ }^{2}$ Division of Rheumatology and Clinical \\ * Corresponding author: takhong.lee@hksh.com
}

Hong Kong Med J 2017;23:419-20

DOI: $10.12809 / \mathrm{hkmj} 176310$

\section{Introduction}

Food-specific immunoglobulin G (IgG) testing is being increasingly used in children to diagnose food allergy and intolerance with often positive results for multiple foods. ${ }^{1}$ Although this enables children to avoid certain food items, there are often no appreciable benefits. The corollary is that patients may eat a food to which they have a true allergy because the IgG test was negative.

It could be argued that avoiding foods to which children are not truly allergic does no harm as long as nutrition is sustained. It is, however, now increasingly accepted that there is nothing to gain in preventing allergies by delaying the introduction of solid foods to infants. ${ }^{2}$ On the contrary, early introduction of solid foods while still breastfeeding might induce tolerance and lower a child's subsequent risk of developing atopic disease. ${ }^{3,4}$ Thus unnecessary avoidance of foods in early life may promote the loss of tolerance and facilitate onset of allergic disease.

In light of this development and the recent experience of a patient who almost died because of misdiagnosis, we feel compelled to remind readers of the published guidelines on IgG testing..$^{5-7}$

\section{The case}

A 3-year-old boy was diagnosed with immediate hypersensitivity to milk at around 8 months of age when he was fed cow's milk formula for the first time. He developed generalised urticaria, vomiting, and appeared to be in respiratory distress. Skin prick testing for cow's milk was positive, confirming his allergy to cow's milk protein. His parents were advised to avoid feeding him any dairy products and to return for regular follow-up to assess the status of his milk allergy. His mother heard about a homeopathic treatment that claimed to eliminate food allergies and hence commenced this treatment. After a number of sessions, she was assured that the boy's food allergy would remit. The mother took the boy for a blood test that purportedly tested for 'intolerance' to 96 food items. This IgG enzyme-linked immunosorbent assay was sent to a laboratory in the US and revealed no sensitivity to cow's milk. The report also indicated that the test had not been evaluated by the Food and Drug Administration (FDA). This should have flashed danger signals as all medical diagnostic tests in the US must be cleared by the FDA. As the test failed to show sensitivity to cow's milk the mother proceeded to give milk to the boy at home whereupon he developed severe anaphylaxis. Unfortunately his adrenaline auto-injector had expired as his mother had defaulted from regular follow-up. He was immediately brought to the emergency department and was resuscitated. Subsequent enquiry revealed that the mother had been informed by the company that the test would not show a positive result if the patient had been avoiding the food, and that the test should be used for 'reference' only. There are many lessons to be learned from this patient's story, not least the danger of relying on the use of unvalidated and unproven tests to diagnose food allergy including the use of IgG.

\section{Authoritative position papers on immunoglobulin $\mathrm{G}$ testing}

The American Academy of Allergy, Asthma \& Immunology states that IgG and IgG subclass antibody tests for food allergy do not have clinical relevance, are not validated, lack sufficient quality control, and should not be performed. ${ }^{2,5,6,8,9}$ The European Academy of Allergy and Clinical Immunology comments that many serum samples show positive IgG4 results without corresponding clinical symptoms. There is a lack of any controlled studies of the diagnostic value of IgG4 testing in food allergy. ${ }^{5}$ The Canadian Society of Allergy and Clinical Immunology also strongly discourages foodspecific IgG testing for the purpose of identifying or predicting adverse reactions to food. ${ }^{6}$

The determination of specific IgG antibodies in serum does not correspond to outcomes of oral food challenge. ${ }^{10}$ There is no evidence that IgG subclasses $^{11}$ or the IgE/IgG4 antibody ratio ${ }^{12}$ are reliable diagnostic tools. In addition, IgG antibodies to common dietary antigens can be detected in health and disease. ${ }^{13}$ In eczema, levels of IgG against a food do not correlate with any clinical 
parameters. $^{7}$ Inappropriate use of the IgG test increases the likelihood of false diagnoses being made with consequent unnecessary dietary restrictions and decreased quality of life. Additionally, and perhaps of greater potential concern, a person with a true IgE-mediated food allergy, who is at significant risk of life-threatening anaphylaxis, may have normal levels of specific IgG to a particular allergen, and may be inappropriately advised to re-introduce this potentially deadly item into their diet. This was precisely the scenario in the patient described above.

\section{Regulation of immunoglobulin G testing}

These types of tests remain in a legal grey zone. In the US, where the majority of such laboratories are found, they remain unregulated as the FDA only has jurisdiction over tests, not laboratories. Individual States are supposed to implement the federal law in banning laboratories from performing non-FDA cleared tests, but many have chosen to ignore this federal law. This has led to a situation where laboratories in 'safe-habour' states continue to accept specimens from other states and from abroad. New York State now prosecutes medical practitioners who refer patient test samples to these out-of-state laboratories. In Hong Kong, an imported pharmaceutical must have regulatory approval in its country of origin before it can be licensed locally. There is no such rule for laboratory tests, however, and this remains a free market for all.

\section{Hong Kong Institute of Allergy's position on immunoglobulin $\mathrm{G}$ testing for diagnosis of food allergy and intolerance}

The strongly held view of the Hong Kong Institute of Allergy (HKIA) is that IgG testing lacks both a sound scientific rationale and evidence of effectiveness. There is a lack of correlation between results and actual symptoms. Even anti-transglutaminase, antideamidated gliadin peptide, and anti-endomysial IgG and IgA are merely antibody markers useful for screening of coeliac disease and monitoring gluten exposure rather than a gold-standard diagnostic modality for this food-related immune-mediated disorder. In the absence of clinical relevance and the potential harm that may result from their use, the HKIA advises against the use of IgG testing for food intolerance, in line with the major allergy and immunology organisations worldwide.

The measurement of food-specific IgG concentrations is of no clinical relevance and should not be part of the diagnostic work-up of food allergy. Instead readers are encouraged to consult explicit recommendations of the HKIA and other authorities ${ }^{5,7,14}$ on how to diagnose and manage food allergies.

\section{Conclusions}

Patients with suspected food-related disorders should seek guidance from a physician for diagnostic testing and interpretation of test results. Measurement of food-specific IgG concentrations to diagnose food allergies and intolerance is strongly discouraged. General practitioners are encouraged to consult their allergy and immunology colleagues if there is any uncertainty about the appropriate management steps for these patients, as unnecessary elimination of foods can lead to severe malnutrition and inappropriate reintroduction of foods can cause serious adverse reactions and possible death.

\section{References}

1. Hon KL, Poon TC, Pong NH, et al. Specific IgG and IgA of common foods in Chinese children with eczema: friend or foe. J Dermatolog Treat 2014;25:462-6.

2. Muraro A, Halken S, Arshad SH, et al. EAACI food allergy and anaphylaxis guidelines. Primary prevention of food allergy. Allergy 2014;69:590-601.

3. Perkin MR, Logan K, Marrs T, et al. Enquiring About Tolerance (EAT) study: Feasibility of an early allergenic food introduction regimen. J Allergy Clin Immunol 2016;137:1477-86.e8.

4. Chan AW, Chan JK, Tam AY, Leung TF, Lee TH. Guidelines for allergy prevention in Hong Kong. Hong Kong Med J 2016;22:279-85

5. Muraro A, Werfel T, Hoffmann-Sommergruber $\mathrm{K}$, et al. EAACI food allergy and anaphylaxis guidelines: diagnosis and management of food allergy. Allergy 2014;69:1008-25.

6. Carr S, Chan E, Lavine E, Moote W. CSACI Position statement on the testing of food-specific IgG. Allergy Asthma Clin Immunol 2012;8:12.

7. Sampson HA, Aceves S, Bock SA, et al. Food allergy: a practice parameter update-2014. J Allergy Clin Immunol 2014;134:1016-25.e43.

8. Johansson SG, Dannaeus A, Lilja G. The relevance of anti-food antibodies for the diagnosis of food allergy. Ann Allergy 1984;53(6 Pt 2):665-72.

9. Bernstein IL, Li JT, Bernstein DI, et al. Allergy diagnostic testing: an updated practice parameter. Ann Allergy Asthma Immunol 2008;100(3 Suppl 3):S1-148.

10. Stiening H, Szczepanski R, von Mühlendahl KE, Kalveram C. Neurodermatitis and food allergy. Clinical relevance of testing procedures [in German]. Monatsschr Kinderheilkd 1990;138:803-7.

11. Kemeny DM, Urbanek R, Amlot PL, Ciclitira PJ, Richards $\mathrm{D}$, Lessof $\mathrm{MH}$. Sub-class of IgG in allergic disease. I. IgG sub-class antibodies in immediate and non-immediate food allergy. Clin Allergy 1986;16:571-81.

12. Jenkins M, Vickers A. Unreliability of IgE/IgG4 antibody testing as a diagnostic tool in food intolerance. Clin Exp Allergy 1998;28:1526-9.

13. Barnes RM. IgG and IgA antibodies to dietary antigens in food allergy and intolerance. Clin Exp Allergy 1995;25 Suppl 1:7-9.

14. Wu A, Chan E, Leung R, Hon E. HKIA guidelines on allergy diagnosis. Available from: http://www.allergy. org.hk/HKIA\%20-\%20Guildelines\%20on\%20Allergy\%20 Diagnosis\%20(Final).pdf. Accessed 18 Apr 2017. 